A N N A L E S Annales de Bretagne et des Pays de l'Ouest

\title{
La tradition manuscrite des " Formules de Tours » et la diffusion des modèles d'actes aux VIIIe et IXe siècles
}

\section{Philippe Depreux}

\section{OpenEdition} Journals

\section{Édition électronique}

URL : http://journals.openedition.org/abpo/1216

DOI : $10.4000 / a b p o .1216$

ISBN : 978-2-7535-1495-9

ISSN : 2108-6443

Éditeur

Presses universitaires de Rennes

Édition imprimée

Date de publication : 20 septembre 2004

Pagination : $57-71$

ISBN : 978-2-7535-0053-2

ISSN : 0399-0826

Référence électronique

Philippe Depreux, «La tradition manuscrite des « Formules de Tours » et la diffusion des modèles d'actes aux VIIIe et IXe siècles", Annales de Bretagne et des Pays de l'Ouest [En ligne], 111-3 | 2004, mis en ligne le 20 septembre 2006, consulté le 01 mai 2019. URL : http://journals.openedition.org/ abpo/1216; DOI : 10.4000/abpo.1216 


\section{II \\ L'abbaye de Saint-Martin et le gouvernement}





\title{
La tradition manuscrite des "Formules de Tours" et la diffusion des modèles d'actes aux VIII et IX ${ }^{\mathrm{e}}$ siècles
}

\author{
Philippe DEPREUX \\ Chargé des études médiévales, \\ Mission historique française en Allemagne (Göttingen) \\ Maître de conférences en histoire médiévale - Université François-Rabelais, Tours \\ Laboratoire de médiévistique occidentale de Paris - LAMOP, UMR 8589 \\ Laboratoire Archéologie et Territoires - CITERES, UMR 6173
}

Les documents qu'on désigne depuis la fin du XIX ${ }^{\mathrm{e}}$ siècle sous le nom de "Formules de Tours " sont des modèles d'actes ${ }^{1}$, dont le texte a été tronqué, parmi lesquels on distingue un corpus de 33 modèles copiés dans plusieurs manuscrits. Les autres modèles d'actes, au nombre de 22 , sont presque exclusivement des documents dont on ne dispose que d'un exemplaire inséré dans telle ou telle copie du recueil ou figurant à la suite du corpus commun (certains des actes ne figurant que dans le manuscrit le plus complet sont annoncés dans la table des matière du codex, d'autres pas); à ces documents s'ajoutent quelques modèles d'actes présentant de grandes similitudes avec les modèles du corpus de référence. On pense que le formulaire de Tours fut réalisé vers le milieu du viII ${ }^{\mathrm{e}}$ siècle ${ }^{2}$ (probablement sous le principat des fils de Charles $\mathrm{Martel}^{3}$ ). Certains actes intéres-

1. Formulae Turonenses vulgo Sirmondicae dictae, dans : Formulae Merowingici et Karolini aevi, p. 128-165 (dorénavant : Form. Turon.).

2. A. GIRY, Manuel de diplomatique, p. 483-484; R. BUCHNER, Die Rechtsquellen, p. 53.

3. K. ZEUMER, «Über die älteren fränkischen Formelsammlungen », p. 63-64. K. Zeumer a proposé de dater la Form. Turon. ${ }^{\circ} 28$ (p. 151), où il est question de l'enfouissement de chartes en terre per timorem illius, qui Turonus civitatem anno presente hostiliter venit et multa mala ibidem perpetravit, de 732 (K. ZEUMER, " Über die älteren fränkischen Formelsammlungen ", p. 61) ou d'environ 763 (introduction à son édition des Form. Turon. : Formulae Merowingici et Karolini aevi, p. 131), année où le comte de Poitiers " se livrait au pillage en Touraine " et fut tué par les homines de l'abbé de SaintMartin, cf. Frédégaire, Chronique..., p. 250-251 (cont., 45). Force est toutefois de se ranger à l'avis de l'éditeur de ces formules, qui se refusait à proposer une datation précise (introduction : Formulae Merowingici et Karolini aevi, p. 131). 
sent directement Saint-Martin de Tours; c'est en particulier le cas du modèle de donation à une église ${ }^{4}$, où il est fait allusion à cette " basilique ", et du modèle d'acte de placement d'un enfant trouvé par les marguilliers de Saint-Martin ${ }^{5}$. Selon Karl Zeumer, cette collection de modèles d'actes, en partie inspirée du formulaire de Marculf, aurait été composée par " un savant connaisseur du droit romain, probablement un notaire ou un greffier, pour son usage personnel ${ }^{6}$ ». De fait, on note un intérêt certain pour le droit romain, en l'occurrence : le droit tel qu'il est transmis par la Lex Romana Visigothorum (autrement dit : cette somme du droit théodosien que représente le Bréviaire d'Alaric). Ce souci de se référer au droit romain n'est pas étonnant - notamment aux marges septentrionales de l'Aquitaine - et la connaissance du droit romain est encore bien attestée, à Saint-Martin de Tours, en plein $\mathrm{X}^{\mathrm{e}}$ siècle $^{7}$. Les réminiscences théodosiennes des "Formules de Tours" prennent tout leur relief lorsqu'on sait que plusieurs des manuscrits en contenant tout ou partie sont de véritables recueils juridiques; il convient à ce propos de rappeler que les « Formules de Tours " ne représentent pas un cas unique : il faut en particulier rapprocher cet état de la tradition manuscrite de celui des "Formules d'Angers ", dont le texte est plus ancien et qui nous sont connues par un seul manuscrit de la fin du VIII ${ }^{\mathrm{e}}$ siècle (copié dans la vallée de la Loire - probablement à Tours ou à Angers), où figure également le Bréviaire d'Alaric ${ }^{8}$. Plusieurs des manuscrits comprenant le Formulaire de Marculf sont également des recueils de lois (qu'il s'agisse de codes de droit romain ${ }^{9}$ ou de la Loi Salique ${ }^{10}$ ). La copie de ce genre de manuscrit plaide en faveur du maintien de notaires publics aux temps carolingiens ${ }^{11}$ et, surtout, en faveur d'une application (ou pour le moins, un souci d'application) du droit écrit par les juges locaux ${ }^{12}$.

Les recueils de formules ont intéressé et intéressent encore en priorité les historiens du droit et des institutions, bien que l'on ait écrit à propos de certains - c'est le cas des "Formules de Flavigny ", dont nous reparlerons - qu'ils ne présentaient d'intérêt que du point de vue de l'histoire littéraire $^{13}$ : les enquêtes portant sur cette documentation relèvent essentiellement des problématiques juridiques, même lorsque des historiens généralistes y portent leur attention, comme c'est par exemple récemment

4. Form. Turon. $\mathrm{n}^{\circ}$ 1, p. 135.

5. Form. Turon. $\mathrm{n}^{\circ} 11$, p. 141.

6. K. ZEUMER, « Über die älteren fränkischen Formelsammlungen », p. 65.

7. J.-P. BRUNTERC'H, " Un monde lié aux archives... ».

8. W. Bergmann, " Die Formulae Andecavenses... ".

9. Leyde, Bibliotheek der Rijksuniversiteit, BPL 114.

10. Paris, Bibliothèque nationale de France, ms. lat. $n^{\circ} 4627$; Leyde, Bibliotheek der Rijksuniversiteit, Voss. Lat. 86.

11. W. BERGMANN, "Fortleben des antiken Notariats... ».

12. R. MCKITTERICK, « Some Carolingian Law-books... »; R. MCKITTERICK, The Carolingians and the written word.

13. R. Schroder et E. V. Kunssberg, Lehrbuch..., p. 294 : « Der Wert der Sammlung ist im wesentlichen nur ein literarhistorischer ". 
le cas d'un historien américain ${ }^{14}$, qui vient de publier un article sur les appennes ou actes relatifs aux chartes perdues ${ }^{15}$, dans lequel il redécouvre l'importance des titres de propriété pour les quidams; les formulaires constituent en effet l'un des rares témoignages sur les actes privés ne faisant pas intervenir un établissement ecclésiastique ${ }^{16}$. Les modèles d'actes tourangeaux, comme les angevins, offrent un observatoire privilégié pour l'étude des subsistances du droit romain aux temps mérovingiens et carolingiens - une question qui obsède les historiens tout comme son négatif, consistant à déceler les éléments d'origine germanique, bien que ces deux manières de lire les sources faussent souvent notre compréhension de la synthèse originale que représentent les institutions médiévales ${ }^{17}$ : parmi divers thèmes que ces recueils de formules permettent d'aborder ${ }^{18}$, des études ont été notamment consacrées aux procédures de déclaration de perte de titres juridiques ${ }^{19}$ et à la conclusion de baux emphytéotiques ${ }^{20}$.

Mon intention n'est pas de me livrer ici à une analyse juridique des "Formules de Tours "; en effet, ces modèles d'actes ne nous présentent, en dépit de leur variété, que certains aspects des pratiques juridiques : gloser telle ou telle formule n'a donc de sens qu'au sein d'une étude plus générale, sur telle ou telle question de droit. Plus intéressante est la question du choix des actes opéré par le notaire : elle nous permettra d'entrevoir le rôle que pouvaient exercer les frères de Saint-Martin dans la société tourangelle de la fin des temps mérovingiens et aux temps carolingiens. Comme on le verra, les manuscrits des "Formules de Tours " datent pour l'essentiel du IX ${ }^{\mathrm{e}}$ siècle (plus généralement, rappelons que les manuscrits par lesquels nous connaissons les formules du monde franc datent tous d'entre le $\mathrm{VIII}^{\mathrm{e}}$ et le $\mathrm{x}^{\mathrm{e}}$ siècle) : un examen de la tradition manuscrite devrait permettre une meilleure appréhension de la raison d'être de ces formulaires - c'est ce à quoi j'aimerais contribuer par cette étude.

\section{Un recueil d'actes}

À l'exception d'un acte de confirmation par le roi des titres détruits par le feu ( $\left.{ }^{\circ} 27\right)$, d'un autre modèle d'appenis, où le détenteur de l'autorité publique à l'origine de ce document n'est pas explicitement désigné ${ }^{21}$, et

14. W. BRown, «The use of norms...»; W. BRown, Unjust seizure...

15. A. GIRY, Manuel de diplomatique, p. 13-15.

16. W. BRown, "When documents are destroyed... ". Cet auteur, qui commente les "Formules d'Angers", ignore apparemment les travaux de W. BERgmann, « Die Formulae Andecavenses... " et de Chr. Lauranson-Rosaz et A. JEannin, "La résolution des litiges... ". Il ignore également la synthèse de B. Tock, "L’acte privé en France ".

17. Ph. DepreuX, " La loi et le droit..." "

18. M. CONRAT, Geschichte der Quellen..., p. 296-297.

19. K. ZEUMER, "Über den Ersatz verlorener Urkunden... "; Chr. LAURANSON-RosaZ et A. JEANNIN, "La résolution des litiges... "; W. BROWN, "When documents are destroyed...".

20. H. BRUNNER, « Die Erbpacht... ».

21. K. ZEUMER, “ Über den Ersatz verlorener Urkunden... », p. 103. 
de quelques actes judiciaires, la majeure partie des documents dont le modèle est transmis dans les "Formules de Tours " sont des actes privés. On observe vaguement un regroupement thématique des actes. Les huit premiers, qui commencent par un modèle de donation à une église, ont trait aux transferts de biens fonciers hors des liens familiaux : procédure d'insinuation, donation, vente, reconnaissance d'usufruit ou précaire. Viennent ensuite quatre actes relatifs aux droits sur les personnes : vente d'esclave ou acte d'asservissement, procès-verbal relatif à la découverte d'un orphelin par les marguilliers de Saint-Martin, acte d'affranchissement. On trouve ensuite un modèle de caution. Puis, viennent douze modèles d'actes ayant trait au droit matrimonial, à la constitution de la dot et aux donations en faveur de parents. À leur suite, on lit un modèle d'acte d'échange et deux formules relatives aux actes perdus. Les cinq modèles suivants concernent le règlement de litiges par voie judiciaire. Parmi les actes copiés seulement dans le manuscrit le plus complet, on trouve, à la suite d'un modèle de prestaire, divers actes relevant des types de documents déjà évoqués et l'un des textes les plus célèbres de cette collection : le modèle de contrat pour " qui se recommande au pouvoir d'un autre ", qui constitue un jalon essentiel dans l'histoire de la vassalité ${ }^{22}$.

Si l'on s'interroge sur l'utilité que les scribes de Saint-Martin pouvaient trouver à copier ces formules, la réponse semble évidente à propos d'un petit nombre seulement de ces actes : il s'agit du modèle de donation à une église $\left(n^{\circ} 1\right)$, du modèle de précaire établi au nom du responsable d'un établissement ecclésiastique $\left(n^{\circ} 7\right)$, du procès-verbal sur les enfants trouvés devant les portes de la basilique $\left(\mathrm{n}^{\circ} 11\right)$ et de la lettre de l'abbé accordant une prestaire $\left(n^{\circ} 34\right)$. En revanche, les actes de constitution de dot ou de divorce n'étaient pas destinés à l'usage monastique. En fait, l'intérêt des scribes était au moins double : d'une part, ils copiaient des actes dont le texte de certains montre qu'ils pouvaient s'avérer directement utiles aux notaires de l'abbaye (c'est le cas, par exemple, de l'acte $n^{\circ} 8$ concernant la vente de biens sis infra terminum sancti illius, dont la conclusion est garantie salvo iure ipsius sancti); d'autre part, il faut admettre que les notaires de Saint-Martin, comme ceux d'autres abbayes, rédigeaient des actes pour les laïcs - ceux qui dépendaient de leur seigneurie ou qui vivaient sur leurs terres (l'acte $n^{\circ} 8$, qu'on vient d'évoquer, affirme éloquemment que les transferts fonciers ne sauraient porter atteinte à la propriété éminente) et les autres. Un modèle d'acte qui figure uniquement dans un manuscrit du IX ${ }^{\mathrm{e}}$ siècle et précède les modèles d'actes concernant les titres perdus des " Formules de Tours " s'avère particulièrement éclairant (Additamentum $\mathrm{n}^{\circ} 7$ ). Il s'agit de la supplique qu'une personne dont les titres ont été détruits lors de l'incendie criminel de sa maison adresse à l'évêque du lieu, à ses vénérables abbés et à ceux qui ont reçu de l'autorité publique la mission de veiller sur la population (vos, qui de parte publica curam vel sollicitudinem habendi positi estis) : cet infortuné leur demande d'intervenir

22. F. L. GANSHOF, Qu'est-ce que la Féodalité?, p. 23-28. 
pour lui en justice si ses biens venaient à être menacés. Ce devoir d'ingérence (ou de protection) pouvait se traduire notamment par le concours des notaires de l'abbaye dans la rédaction des chartes de ces personnes. La présence d'actes visant au règlement des différends s'explique probablement aussi par l'intervention possible de l'abbé, comme arbitre ou comme juge, ou par le concours des notaires de l'abbaye au tribunal public.

Reste un acte isolé, copié plus loin dans le manuscrit le plus complet, à la suite de textes canoniques et du modèle (regula formatarum) attribué au patriarche de Constantinople, Atticus ${ }^{23}$ (406-425). Il s'agit d'un témoignage précieux de la cure des âmes dont Saint-Martin de Tours avait la responsabilité. Ce modèle d'epistola formata (Additamentum $\mathrm{n}^{\circ} 8$ ), date de 815834. L'archevêque de Tours (en l'occurrence : Landramne, 815-836) atteste qu'un prêtre avait été ordonné par son prédécesseur, Joseph (792-815), à la demande de l'abbé Fridugise (804-834), in titulo sancti Martini, in villa qui dicitur illa, et qu'il avait lui-même procédé à son examen. C'est pourquoi l'archevêque s'adresse à tel évêque, pour qu'il soit permis à ce prêtre d'accomplir son office dans le diocèse du destinataire. On a ainsi la preuve de l'exercice que faisait Saint-Martin de Tours du droit de présentation, concernant des églises de domaines sis hors du diocèse de Tours.

\section{Les manuscrits}

Les " Formules de Tours " nous sont transmises par neuf manuscrits, dont cinq contiennent presque intégralement le corpus constitué des 33 premiers modèles d'actes et quatre les contiennent partiellement - voire n'en contiennent que très peu. Plusieurs d'entre eux comptent parmi les manuscrits que Rosamond McKitterick, développant l'intuition de Bernhard Bischoff, attribue à un scriptorium très proche du pouvoir impérial ayant copié des manuels juridiques en série et qui serait celui de Saint-Martin de Tours ${ }^{24}$.

Le manuscrit de référence est un codex copié à Tours dans la première moitié du IX ${ }^{\mathrm{e}}$ siècle et actuellement conservé à Varsovie ${ }^{25}$. Ce manuscrit comprend le Bréviaire d'Alaric, la Loi Salique, le décret de Childebert II (596) et les "Formules de Tours" (copiées par une seule main ${ }^{26}$ ) comprenant le corpus des 33 premiers modèles complété de treize actes (douze selon la numérotation du manuscrit ${ }^{27}$ ) et des modèles de litterae formatae

23. Cette attribution est erronée, cf. C. FABRICIUS, « Die Litterae formatae... », p. 52-53.

24. R. MCKITTERICK, The Carolingians and the written word, p. 57-58; R. MCKITTERICK, "Zur Herstellung von Kapitularien... ".

25. Varsovie, Biblioteka Uniwersytecka, 1. Description dans : H. MoRDEK, Bibliotheca capitularium..., p. 898-903. Je n'ai malheureusement pas pu voir ce manuscrit, contrairement aux manuscrits parisiens dont il sera question plus loin.

26. Introduction à l'édition de K. Zeumer : Formulae Merowingici et Karolini aevi, p. 131.

27. Les Form. Turon. n 39 et 40 sont fondues en un seul texte, cf. K. ZeUmER, « Über die älteren fränkischen Formelsammlungen ", p. 52 et le tableau synoptique infra p. 68-69. 
mêlés à des textes canoniques; on y trouve aussi des extraits du livre $\mathrm{v}$ des Etymologies d'Isidore de Séville, consacré aux lois. Il s'agit donc d'un recueil cohérent, que Karl Zeumer considérait comme un manuel juridique destiné à l'usage courant d'un juriste tourangeau ayant copié les " Formules de Tours " directement sur le recueil originel ${ }^{28}$. La langue y est meilleure que dans les autres manuscrits : Karl Zeumer y reconnaissait un produit de la "renaissance " carolingienne ${ }^{29}$ dont il entendait gommer les corrections grâce aux leçons des autres manuscrits, pour tenter de rétablir ce qu'il considérait comme la version originelle ${ }^{30}$.

Un autre manuscrit s'avère très proche du manuscrit de Varsovie : il s'agit du manuscrit latin $n^{\circ} 4409$ de la Bibliothèque nationale de France, copié vers la fin du IX ${ }^{\mathrm{e}}$ siècle en France du Nord ${ }^{31}$. Ce manuscrit comprend exclusivement des textes juridiques; son contenu est assez similaire à celui du manuscrit de Varsovie. Il comporte l'intégralité du corpus des 33 actes, à ceci près qu'il ne compte que 32 rubriques (deux actes étant fondus en un seul ${ }^{32}$ ). Un manuscrit du Bréviaire d'Alaric copié au $\mathrm{x}^{\mathrm{e}}$ siècle, actuellement conservé au Vatican ${ }^{33}$, dans lequel ne se trouve qu'une douzaine d'actes tourangeaux, porte la même particularité de numérotation, ce qui permet de penser que l'origine en est la même. Le manuscrit latin $n^{\circ} 4405$ de la Bibliothèque nationale de France, qui date du $\mathrm{IX}^{\mathrm{e}}$ siècle, est également un recueil juridique de droit romain; seulement deux modèles d'actes tourangeaux y figurent, à la suite d'une formule originale qu'on ne trouve que dans ce manuscrit ${ }^{34}$. Par ailleurs, quatre formules élaborées directement d'après les modèles d'actes tourangeaux figurent à la fin d'un manuscrit copié en Francie occidentale vers le milieu du IX ${ }^{\mathrm{e}}$ siècle et ayant appartenu à Saint-Martial de Limoges ${ }^{35}$. Il s'agit là encore d'un recueil de lois et de textes juridiques.

Certains manuscrits des "Formules de Tours " ne sont, contrairement aux autres, pas des recueils juridiques, mais des manuscrits composites comprenant entre autres choses le Formulaire de Marculf; un manuscrit, conservé à Copenhague, est uniquement un recueil de formules (Kongelige Bibliotek, Coll. Fabr. 84). Le manuscrit latin $n^{\circ} 10756$ de la Bibliothèque nationale de France, un assemblage de plusieurs manuscrits dont les folios contenant les "Formules de Tours " furent copiés au IX ${ }^{\mathrm{e}}$ siècle, comprend

28. K. ZEUMER, « Über die älteren fränkischen Formelsammlungen », p. 59.

29. Ph. DepreuX, "Ambitions et limites des réformes culturelles".

30. K. ZEUMER, «Über die älteren fränkischen Formelsammlungen », p. 58 : « Es bleibt nur die Möglichkeit, den Text des Cod. Vars. aus den anderen Handschriften zu verbessern, um die Vorlage desselben möglichst wiederherzustellen; denn das muss unser Ziel sein ".

31. Description dans : H. MoRDEK, Bibliotheca capitularium..., p. 463-466.

32. Form. Turon. $\mathrm{n}^{\circ} 2$ et 3 .

33. Biblioteca Apostolica Vaticana, Reg. lat. 852.

34. Add. $\mathrm{n}^{\circ} 7$ + Form. Turon. $\mathrm{n}^{\circ} 27$ et 28 (ces modèles d'actes ne sont pas numérotés dans le manuscrit; ils sont introduits par l'expression : alium relatum).

35. Cité du Vatican, Biblioteca Apostolica Vaticana, Reg. Lat. 1050; description dans : H. МоRDEK, Bibliotheca capitularium..., p. 847-852. 
32 des 33 actes du corpus de référence ${ }^{36}$; à l'instar du manuscrit de Varsovie, la table des matières se poursuit au-delà des titres de ce corpus, en présentant certaines divergences avec la table des matières du manuscrit d'origine tourangelle (l'ordre n'y est pas tout à fait le même et certains titres sont nouveaux; on y trouve l'annonce d'actes correspondant à ceux du Formulaire de Marculf). Karl Zeumer avait avoué son impuissance à démêler les fils de la tradition manuscrite de ces textes ${ }^{37}$. Retenons pour l'instant que l'état dans lequel les diverses versions des "Formules de Tours " nous sont parvenues ne peut s'expliquer que par l'existence de manuscrits intermédiaires aujourd'hui disparus.

Le manuscrit des " Formules de Tours " probablement le plus ancien, c'est-à-dire le manuscrit latin $n^{\circ} 2123$ de la Bibliothèque nationale de France, copié entre 795 et 816 selon Wallace Lindsay ${ }^{38}$ (avec des additions des $\mathrm{x}^{\mathrm{e}}$ et $\mathrm{XI}^{\mathrm{e}}$ siècles), comprend des textes conciliaires et des extraits du Liber pontificalis et d'œuvres de divers auteurs; le recueil de modèles d'actes qu'on y lit est connu sous le nom de "Formules de Flavigny " : il s'agit d'une compilation que Karl Zeumer datait du viII ${ }^{\mathrm{e}}$ ou du $\mathrm{IX}^{\mathrm{e}}$ siècle (avec une préférence pour une datation haute ${ }^{39}$ ); cette compilation fut réalisée principalement à partir du Formulaire de Marculf et des "Formules de Tours". Ce recueil, en raison de sa structure, ne correspond pas directement à l'archétype que Karl Zeumer voulait restituer; en revanche, il me semble fondamental du point de vue de l'histoire de ces formules et de leur réception. Il convient, pour compléter le tableau de la tradition manuscrite des "Formules de Tours ", de mentionner encore un manuscrit du Vatican (Reg. Lat. 612), qui contient les "Formules de Merkel " - il en sera à nouveau question à propos de l'abbaye de Flavigny.

\section{Saint-Martin de Tours et Flavigny}

Comme on vient de le voir, les "Formules de Tours " ont été utilisées pour la réalisation d'un formulaire largement inspiré de celui de Marculf et réalisé à Flavigny. Comment cela fut-il possible? Il convient, dans le cadre de ce colloque, de s'interroger sur le rôle qu'Alcuin put éventuellement jouer en la matière. En effet, Alcuin, abbé de Saint-Martin de Tours,

36. Form. Turon. $\mathrm{n}^{\circ} 31$ manque. Apparemment, le modèle de ce manuscrit comprenait un item supplémentaire, puisque le dernier acte de cette collection porte le numéro XXXIV.

37. K. ZEUMER, " Über die älteren fränkischen Formelsammlungen », p. 51-53 (avec tableau synoptique).

38. W. LindSAY, Notae Latinae..., p. 472.

39. L'éditeur des Formulae est revenu sur la datation qu'il avait proposée dans K. ZEUMER, " Über die älteren fränkischen Formelsammlungen ", p. 68; dans l'introduction à son édition (Formulae Merowingici et Karolini aevi, p. 471), il se montre assez prudent, tout en exprimant une préférence pour le viII ${ }^{\mathrm{e}}$ siècle : Quae cum ita sint, collectionem formularum iam saeculo vIII. compositam esse proprius vero esse videtur, quam ut saeculo demum IX. hoc factum sit. R. BUCHNER, Die Rechtsquellen, p. 53, date cette collection du $3^{\mathrm{e}}$ quart du viII ${ }^{\mathrm{e}}$ siècle sans se justifier. 
fut également abbé de Flavigny ${ }^{40}$ d'environ 797 jusqu'à sa mort ${ }^{41}$, en 804 . Rien ne permet d'établir avec certitude qu'il est à l'origine de la diffusion du recueil d'actes tourangeau en Bourgogne; cette hypothèse, à ma connaissance, est inédite : dans son étude sur le scriptorium de Flavigny, Jean Marilier n'évoque pas cette possibilité ${ }^{42}$, qui me semble avoir pour elle quelque degré de probabilité. Eu égard aux liens qu'entretenait l'abbaye de Flavigny avec le pouvoir central depuis le temps de Charles Martel $^{43}$, les moines jouissaient certes d'un réseau suffisamment important pour se procurer un exemplaire des "Formules de Tours" et les adapter à leurs usages bien avant d'être soumis à l'abbé de Saint-Martin de Tours ${ }^{44}$. Comme exemples d'adaptation du formulaire aux traditions locales ou aux besoins des scribes bourguignons, citons l'introduction d'un modèle de testament d'après celui du fondateur de Flavigny, Widerad $^{45}$, ou l'apposition du vocable finis, en usage en Bourgogne, à celui de condita, caractéristique de la France de l'Ouest ${ }^{46}$. Si l'hypothèse selon laquelle Alcuin se trouverait à l'origine de la rédaction des "Formules de Flavigny " pouvait être prouvée, on disposerait - à l'instar de l'introduction en Bavière des "Formules de Lindenbrog " par l'abbé de Saint-Amand, Arn, lorsqu'il devint évêque de Salzbourg ${ }^{47}$ - d'un exemple supplémentaire de diffusion, par un abbé puissant, d'un formulaire dans un (ou plusieurs) monastère(s) soumis à son autorité - avec, peut-être, le dessein d'uniformiser la rédaction des actes et la formation dispensée aux scribes, tout en respectant certaines particularités locales (même si Martina Hartmann inclinait autrefois à interpréter le peu d'informations dont nous disposons sur l'action d'Alcuin en tant qu'administrateur des biens monastiques comme l'expression du fait que ce genre de problème s'avérait loin d'être sa tasse de thé ${ }^{48}$; un examen centré sur les sources tourangelles l'a toutefois conduite à revoir son jugement ${ }^{49}$ ). Peut-on aller plus loin, et identifier l'un des scribes qui copièrent le manuscrit latin 2123 ? On y lit, au

40. Alcuin succéda à Zacho, ordonné en 793 (Annales Flaviniacenses seu Laudonenses, MGH SS 3, p. 151), qui décéda lors de la quatrième année de son abbatiat (Series abbatum Flaviniacensium, MGH SS 8, p. 502); Apollinaire, le successeur d'Alcuin, reçut la charge abbatiale de la part de Charlemagne sept ans plus tard (ibid.). Selon E. HLAWITSChKA, "Textkritisches... ", l'auteur de la liste des abbés serait Hugues de Flavigny.

41. Hugues de Flavigny, Chronicon, MGH SS 8, p. 352.

42. J. MARILIER, "Le scriptorium de l'abbaye de Flavigny... ". Je remercie Thomas Lienhard de l'obligeance avec laquelle il m'a fait parvenir une copie de cet article.

43. J. SEMmLER, " Die Aufrichtung der karolingischen Herrschaft... ", p. 26-27.

44. C'est en 796 qu'Alcuin fut nommé abbé de Tours, cf. A. KLEINCLAUSZ, Alcuin, p. 157;

J. ChelinI, "Alcuin, Charlemagne et Saint-Martin..." ".

45. U. Nonn, " Merowingische Testamente... ", p. 33-34.

46. Additamentum $\mathrm{n}^{\circ} 3$ aux "Formules de Flavigny (Formulae Merowingici et Karolini aevi, p. 490); à ce propos, cf. R. SCHRODER, " Über die fränkischen Formelsammlungen ", p. $86-87$.

47. R. SCHRODER, "Über die fränkischen Formelsammlungen », p. 94-111.

48. M. StRATMANN, "Schriftlichkeit in der Verwaltung... ", p. 271-272.

49. Cf. sa contribution au présent volume, infra p. 91-102. 
$\mathrm{f}^{\circ}$ 91, la mention : Walefredus me fecit ${ }^{50}$. Il se trouve que l'un des moines de Saint-Martin du temps de l'abbé Fridugise mentionnés dans le livre de fraternité de l'abbaye de Saint-Gall porte le même nom (Walafredus ${ }^{51}$ ) ce nom ne figure en revanche pas dans la liste des frères de Flavigny du livre de fraternité de la Reichenau ${ }^{52}$. On pourrait donc imaginer que ce Walefredus était un moine de Saint-Martin ayant effectué un séjour à Flavigny du temps d'Alcuin. On sait que les noms de quatre scribes de Saint-Martin figurent dans la liste des frères tourangeaux envoyée aux moines de Saint-Gall ${ }^{53}$. En contient-elle un cinquième - en l'occurrence, celui de Walefredus? La chose relève du possible, voire du probable, mais malheureusement pas du certain.

Le manuscrit latin $n^{\circ} 2123$ de la Bibliothèque nationale de France n'est pas le manuscrit originel de ce mélange du Formulaire de Marculf et des "Formules de Tours " que forment les "Formules de Flavigny ". On dispose en effet d'un autre manuscrit du IX ${ }^{\mathrm{e}}$ siècle, conservé à Copenhague (Kongelige Bibliotek, Coll. Fabr. 84), dont la structure est telle qu'elle ne s'explique que par l'existence d'une bifurcation dans la tradition manuscrite; le codex où figurait la collection originelle est perdu. Le manuscrit de Copenhague présente le double intérêt d'être exclusivement un recueil de formules et de témoigner d'un réel souci d'efficacité de la part du scribe à l'origine de ce recueil, puisqu'il regroupa certains actes ou élimina certains documents redondants ${ }^{54}$.

Selon Richard Schröder, Flavigny pourrait également être l'abbaye où furent composées les "Formules de Merkel ", connues par un manuscrit du Vatican (Reg. Lat. 612) datant de la fin du IX ${ }^{\mathrm{e}}$ siècle ${ }^{55}$. Eu égard à la nature des textes qu'il contient, ce manuscrit composite ${ }^{56}$ pourrait également avoir été copié en Touraine ou dans le diocèse de Paris ${ }^{57}$; force est de reconnaître qu'on ne parvient pas à établir son origine ${ }^{58}$. Le formulaire de Merkel peut être décomposé en trois parties : un premier corpus d'actes privés fut réalisé sous Charlemagne ${ }^{59}$; il fut complété au cours du IX ${ }^{\mathrm{e}}$ siècle par des actes de natures variées ${ }^{60}$ et $s$ 'achève par un recueil de modèles

50. Paris, Bibliothèque nationale. Catalogue général des manuscrits latins, tome 2, éd. Philippe LAUER, Paris 1940, p. 329-330.

51. Libri confraternitatum sancti Galli, p. 13.

52. Ibid., p. 225.

53. O. G. OEXLE, Forschungen..., p. 42-43. On dispose d'une mise en perspective historique de la liste des frères de Saint-Martin grâce à W. VogLER, "St. Martin in Tours und St. Gallen..." ".

54. K. ZEUMER, " Die Lindenbruch'sche Handschrift... ".

55. R. SCHRODER, " Über die fränkischen Formelsammlungen », p. 86-87.

56. Description dans : J. MERKEL, " Ein westfränkisches Formelbuch ", p. 194-195.

57. Introduction à l'édition de K. Zeumer : Formulae Merowingici et Karolini aevi, p. 240.

58. Capitula episcoporum, tome 2, p. 124.

59. G. WAITZ, « Über die Merkelschen Formeln », p. 533-541.

60. Parmi ces actes, le $n^{\circ} 44$ correspond à un modèle assez répandu, qu'on trouve dans les "Formules impériales" $\left(\mathrm{n}^{\circ} 33\right)$ et ailleurs (Formulae extravagantes $\mathrm{I}, \mathrm{n}^{\circ} 17$ : Formulae Merowingici et Karolini aevi, p. 544). 
épistolaires ${ }^{61}$. Le notaire à l'origine de la première partie de cette collection s'est inspiré du Formulaire de Marculf, mais aussi de quelques "Formules de Tours ". À cet égard, il faut noter que le manuscrit dont disposait l'auteur était soit le manuscrit de Varsovie, soit un manuscrit, aujourd'hui perdu, appartenant à la même filiation manuscrite que ce codex dont on sait toutefois qu'il devait se trouver à Langres au XI ${ }^{\mathrm{e}}$ siècle ${ }^{62}$; en effet, l'une des «Formules de Merkel " $\left(n^{\circ} 9\right)$ correspond à un modèle d'acte figurant dans le manuscrit de Varsovie alors même qu'il n'est pas répertorié dans la liste des chapitres initiale (Additamentum $\mathrm{n}^{\circ} 2$ ). Cette observation vient donc renforcer la probabilité d'une réalisation de ce recueil de formules en Bourgogne. Si tel était le cas, on disposerait d'un indice supplémentaire concernant la diffusion de modèles d'actes de Saint-Martin de Tours à l'extérieur de la Touraine.

\section{Les " Formules de Tours" dans les courants d'échanges carolingiens}

Contrairement aux «Formules impériales ", dont l'unique manuscrit ${ }^{63}$ ne peut pas être considéré comme un manuel, puisque les actes sont disséminés en plusieurs endroits du codex et sont, par conséquent, d'une consultation malaisée (ce qui ne veut pas dire que les scribes ne l'utilisaient pas, ainsi que le suggère la présence d'un modèle d'acte provenant des " Formules impériales " dans un manuscrit juridique copié à Tours durant le deuxième quart du IX ${ }^{\mathrm{e}}$ siècle ${ }^{64}$ ), la présentation des "Formules de Tours " à la suite d'une table des matières, copiée avec des variantes dans les cinq manuscrits les plus complets, prouve le même souci d'utilité qu'on observe chez Marculf, dont le formulaire était connu de l'auteur du recueil tourangeau. L'érudition technique des scribes des "Formules de Tours " se traduit par l'usage partiel de notes tironiennes; il s'agit-là d'un mode d'écriture bien maîtrisé à Tours, comme en témoignent d'ailleurs éloquemment le recueil des «Formules impériales " et d'autres manuscrits tourangeaux ${ }^{65}$.

On ne dispose pas, concernant les " Formules de Tours ", d'étude systématique sur l'influence qu'elles ont pu exercer sur la rédaction des actes de la pratique ${ }^{66}$, à l'instar de ce qui a pu être établi pour le Formulaire de Marculf ou les "Formules de Lindenbrog " vers la fin des temps mérovingiens et aux temps carolingiens ${ }^{67}$. Quant à l'influence que ces modèles

61. Sur ce genre documentaire, cf. G. CONSTABLE, Letters and Letter-collections.

62. H. MORDEK, Bibliotheca capitularium..., p. 899 et 902.

63. Paris, Bibliothèque nationale de France, ms. latin $n^{\circ} 2718$.

64. Paris, Bibliothèque nationale de France, ms. latin nouv. acq. 204; cf. B. KRusch, Die Lex Bajuvariorum..., p. 105-106 (il s'agit du n ${ }^{\circ} 15$ des «Formules impériales »). Description de ce manuscrit dans : H. MORDEK, Bibliotheca capitularium..., p. 621-624.

65. D. GANZ, "On the History of Tironian Notes".

66. Sur l'utilisation du formulaire de Tours à Redon, cf. le commentaire d'Hubert Guillotel dans : Cartulaire de l'abbaye Saint-Sauveur de Redon, Rennes, Amis des Archives historiques du diocèse de Rennes, Dol et Saint-Malo, 1998, p. 18.

67. H. ZatscheK, « Die Benutzung der Formulae Marculfi... ". 
d'actes ont pu avoir sur la rédaction des chartes au sein même de l'abbaye tourangelle, Pierre Gasnault observe " un rapport entre les plus anciens actes qui nous sont parvenus et les recueils de formules mérovingiennes ", mais " à partir du IX ${ }^{\mathrm{e}}$ siècle, l'influence des formulaires ne se fait plus sentir. À cette époque, les rédacteurs se reportent aux actes que renfermait déjà le chartrier pour se guider dans la rédaction d'actes nouveaux ${ }^{68}$ ". Eu égard au rayonnement de l'établissement dans lequel il fut élaboré, il convient de s'interroger, plus largement, sur la place qu'occupe ce formulaire dans les courants d'échanges aux temps carolingiens.

À ce propos, on a supposé un rapport entre les «Formules de Tours " et la Loi Romaine de Coire; cette question s'avère particulièrement délicate. Karl Zeumer avait déjà observé quelques similitudes entre certains modèles d'actes ligériens et cet Epitome du Bréviaire d'Alaric qui semble avoir été réalisé en Rhétie au cours de la première moitié du VIII ${ }^{\mathrm{e}}$ siècle ${ }^{69}$ et présente l'intérêt - à la différence des autres "résumés " du Bréviaire - de se vouloir " une législation actuelle et pratique ", selon la formulation de Jean Gaudemet ${ }^{70}$. Tous les parallèles qu'on a pu relever ${ }^{71}$ ne sont pas significatifs. Ainsi, il convient de relativiser l'observation faite par Elisabeth MeyerMarthaler d'une référence possible de la Loi Romaine de Coire à l'acte n ${ }^{\circ} 11$ des "Formules de Tours " ou au n ${ }^{\circ} 49$ des «Formules d'Angers" (elle évoque d'ailleurs la possibilité d'une référence à un autre recueil similaire ${ }^{72}$ ). En particulier, l'exposition de l'enfant dans l'église ou sur le parvis, mentionnée dans ce texte juridique ${ }^{73}$ et dans les formules en question, ne saurait prouver une influence directe, puisqu'on trouve le même cas de figure à Trèves, par exemple, comme l'illustre une anecdote relatée dans les Miracles de saint Goar $^{74}$. En revanche, il convient de souligner que les mesures financières prises pour entretenir un orphelin qu'on a recueilli sont censées être destinées à le nutrire à la fois dans le modèle d'acte tourangeau concernant l'accueil d'un tel enfant ${ }^{75}$ et dans le passage de la Loi Romaine de Coire correspondant ${ }^{76}$. Ce qui donne à cette observation quelque intérêt réside en ce que, apparemment, cet Epitome est le seul des " résumés " du Bréviaire à receler cette formulation ${ }^{77}$. Une telle adaptation aux usages de l'époque n'est pas pour étonner lorsqu'on sait que des termes du droit franc, tels fredus ou admallare, figurent dans ce texte juridique ${ }^{78}$. Le nombre des coïnci-

68. P. GaSnAult, "Les actes privés... ", p. 29-30.

69. Lex Romana Curiensis, p. XLVI-LII.

70. J. Gaudemet, Le Bréviaire d'Alaric..., p. 50.

71. M. CONRAT, Geschichte der Quellen..., p. 297.

72. E. MEYER-MARTHALER, Römisches Recht in Rätien..., p. 18.

73. Lex Romana Curiensis, p. $219: \mathrm{v}, 7$.

74. J. Boswell, The kindness of strangers..., p. 218; sur l'interprétation de cet épisode, cf. Ph. DepreuX, Les sociétés occidentales..., p. 59.

75. Form. Turon. $\mathrm{n}^{\circ} 24$.

76. Lex Romana Curiensis, p. 155 : III, 19, 4.

77. M. ConRat, Geschichte der Quellen..., p. 297 n. 2; cf. la présentation synoptique dans : Lex Romana Visigothorum, p. 100-101.

78. R. Buchner, Die Rechtsquellen, p. 37. 
dences nous autorise à penser que le notaire tourangeau et le praticien du droit de Rhétie se référaient aux mêmes pratiques; de là à prétendre que l'un de ces deux textes à peu près contemporains a pu servir de modèle pour la rédaction de l'autre, il y a un pas que l'on ne peut franchir, faute de preuve manifeste.

Les contacts personnels entre les membres de la communauté de SaintMartin et ceux des grands établissements alémaniques que sont la Reichenau et Saint-Gall sont attestés dès le règne de Charlemagne ${ }^{79}$. Il existe également un indice plus ancien des relations possibles entre les praticiens du droit de ces deux régions. On conserve en effet le texte d'un acte de vente par lequel, le 28 juin 733, un certain Gundoson vendit à l'abbé de SaintMartin de Tours ce qu'il possédait par héritage et avait acquis en Alémanie (dans le Briesgau), à Kandern (sur le fleuve Kander ${ }^{80}$, au sud-ouest de la Forêt Noire). Comme le note Pierre Gasnault à propos de cet acte, "il est difficile de juger s'il a eu pour modèle la formule 2 " (publiée dans les additamenta, c'est-à-dire dans le modèle de vinditio ad ecclesiam qu'on trouve dans le manuscrit de Varsovie mais qui n'est pas annoncé dans la table des matières de ce codex) " ou si, au contraire, cette charte a servi de modèle à la formule ". Si l'on s'en tient à la datation des "Formules de Tours " proposée par Karl Zeumer et rappelée au début de cette étude, il semblerait que ce soit l'acte qui se trouve à l'origine du modèle, et non l'inverse (la formule du manuscrit de Varsovie n'est pas complète; la fin de l'acte de 733 présente par ailleurs des similitudes avec celle de la formule $n^{\circ} 9$ « de Merkel ", elle-même similaire à la formule du manuscrit de Varsovie). Cet acte de vente ne constitue pas le seul témoignage d'une emprise de SaintMartin en Alémanie ${ }^{81}$, qui n'est pas sans rappeler l'attitude de l'abbé de Saint-Denis au vIII ${ }^{\mathrm{e}}$ siècle, à la faveur de la politique d'expansion des Pippinides ${ }^{82}$. C'est d'ailleurs le même jour (le 31 août 790 ), alors qu'il se trouvait aux portes de Mayence (à Kostheim), que Charlemagne restitua à Saint-Denis et à Saint-Martin des biens confisqués du temps de Pépin le Bref et de Carloman : ces biens étaient passés au pouvoir de quidams avant d'être vendus à ces monastères, qui avaient été contraints de les restituer au roi; ce dernier, par des diplômes presque similaires, les leur rendit gracieusement $^{83}$. Dans le cas de Saint-Martin, il s'agit de biens que l'Alaman Fulridus détenait dans le Breisgau, à Steinenstadt (sur le Rhin, au sud-ouest de Müllheim). Ce diplôme et l'acte de vente de 733 ne nous mènent certes pas jusqu'en Rhétie, mais prouvent pour le moins l'intérêt de Saint-Martin

79. W. VoGLER, «St. Martin in Tours und St. Gallen... », p. 12.

80. Gallia Christiana in provincias ecclesiasticas distributa..., tome 14, éd. B. HAUREAU, Paris 1856, instrumenta $\mathrm{n}^{\circ} 4$, col. 6-7.

81. Hélène NoIzET, Les relations de Saint-Martin de Tours avec ses possessions à l'époque carolingienne, mémoire de maîtrise sous la direction de H. Galinié, Université de Tours, 1997, p. 23, juge ce phénomène négligeable.

82. J. Fleckenstein, « Fulrad von Saint-Denis... »; A. Stoclet, Autour de Fulrad...

83. Die Urkunden der Karolinger, tome 1, p. 224-225 (diplômes nº 166 et 167, respectivement pour Saint-Denis et pour Saint-Martin). 
pour cette partie du monde carolingien en marge de sa zone naturelle d'influence et la plausibilité de la circulation de documents tourangeaux dans ces régions orientales.

Cette étude permet de souligner l'importance du scriptorium de SaintMartin de Tours, évoquée jusqu'ici principalement à propos de la production de Bibles et autres manuscrits enluminés ${ }^{84}$, de Martinelli $^{85}$ et de recueils juridiques ${ }^{86}$. Force est en effet de constater que la reconstitution de la tradition manuscrite des " Formules de Tours " suppose l'existence de manuscrits aujourd'hui perdus et devrait porter à au moins environ quatorze le nombre des manuscrits ayant contenu tout ou partie de cette collection (outre l'archétype, il me semble en effet qu'on doit supposer l'existence d'un manuscrit intermédiaire ${ }^{87}$ entre ce dernier et le manuscrit de Varsovie et un autre intermédiaire ${ }^{88}$ entre la version originelle et le manuscrit parisien $n^{\circ} 10756$; par ailleurs, Karl Zeumer avait supposé l'existence d'un manuscrit intermédiaire entre l'archétype des " Formules de Flavigny ", lui-même dérivé de celui des "Formules de Tours " ou d'une copie, et le manuscrit de Copenhague, dont la tradition est indépendante du manuscrit de Paris ${ }^{89}$ ). Tous ces manuscrits ne furent assurément pas produits à Tours (quatre ou cinq tout au plus ont ou devraient avoir été produits à Tours, trois ou quatre à Flavigny, et le reste dans la partie septentrionale de la Francie occidentale - ce qui n'exclut pas une production plus importante du scriptorium de Tours). Par conséquent, la politique de diffusion de ces formules ou les dispositions favorables à leur réception de la part de tiers s'avèrent peut-être tout aussi importantes que la copie de manuscrits à Saint-Martin.

Pour mieux apprécier la diffusion des "Formules de Tours ", il conviendrait de reconstituer l'histoire de chacun des manuscrits et de préciser ses localisations successives durant le haut Moyen Âge, puisque c'est à cette époque seulement qu'on éprouvait le besoin de copier ces formules qui disparaissent ensuite pour prendre un nouveau jour au Moyen Âge central. Une telle enquête est rarement possible. Il conviendrait par ailleurs de jauger l'efficacité d'une éventuelle volonté de diffuser ces formules à l'aune de la diffusion de l'ensemble des formulaires et autres manuscrits contenant des modèles d'actes. En comparaison avec les autres recueils, la collection tourangelle fut copiée relativement souvent : pour ne prendre que quelques exemples parmi certains des formulaires les plus anciens, rap-

84. P. GaSnAUlt, "Le scriptorium de Saint-Martin... ".

85. P. Bourgain et M. Heinzelmann, "L'œuvre de Grégoire de Tours... ».

86. R. MCKITTERICK, "Some Carolingian Law-books..." "

87. C'est dans ce manuscrit que devaient se trouver la liste des chapitres qu'on lit dans le manuscrit de Varsovie et le texte des actes annoncés sous les numéros 35 et 36.

88. C'est dans ce manuscrit que devaient se trouver la liste des chapitres qu'on lit dans le manuscrit $n^{\circ} 10756$ de la Bibliothèque nationale de France, le texte des actes correspondants et celui des actes figurant en lieu et place du $n^{\circ} 31$.

89. K. ZEUMER, « Die Lindenbruch'sche Handschrift... », p. 600. 


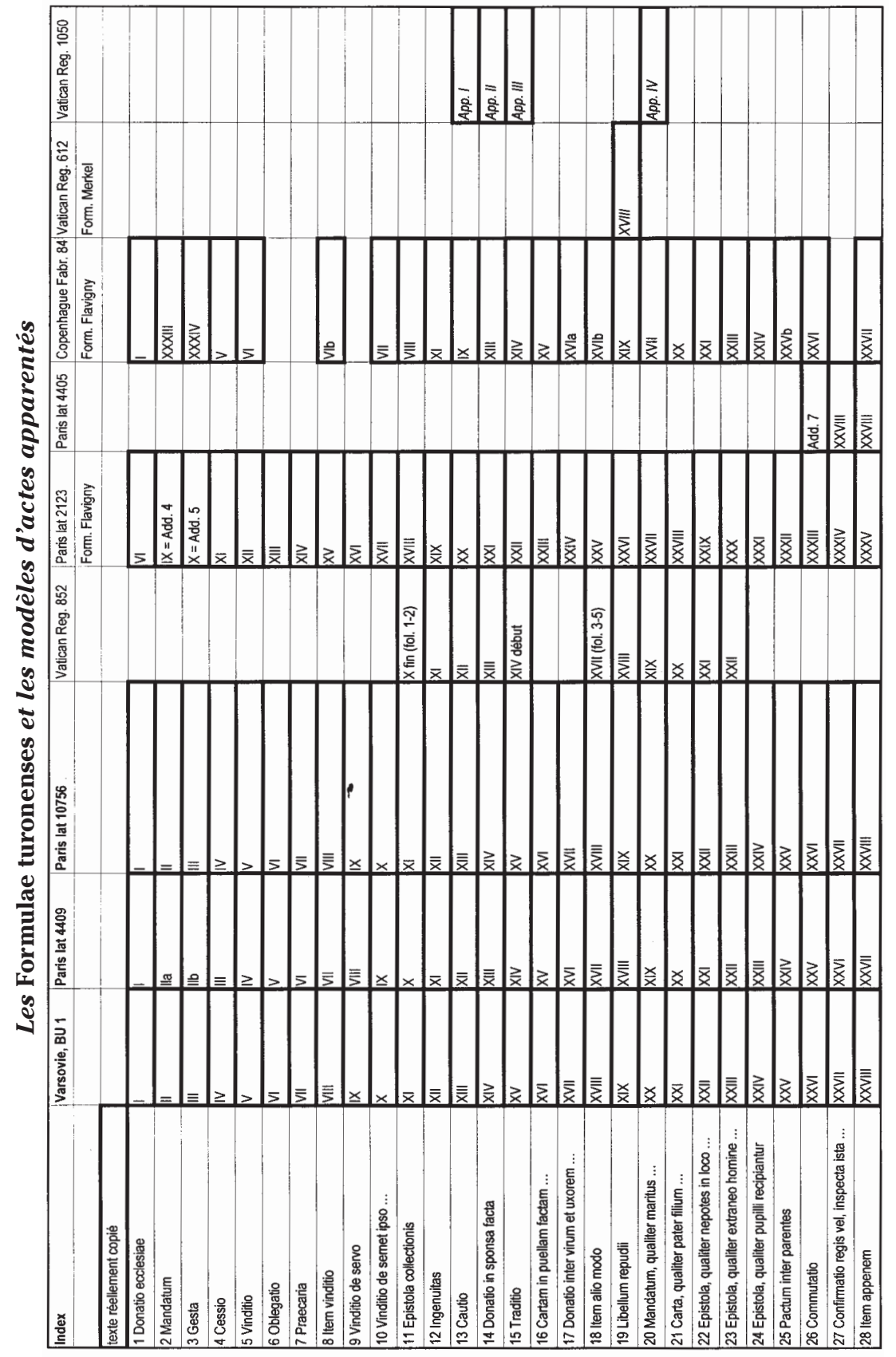




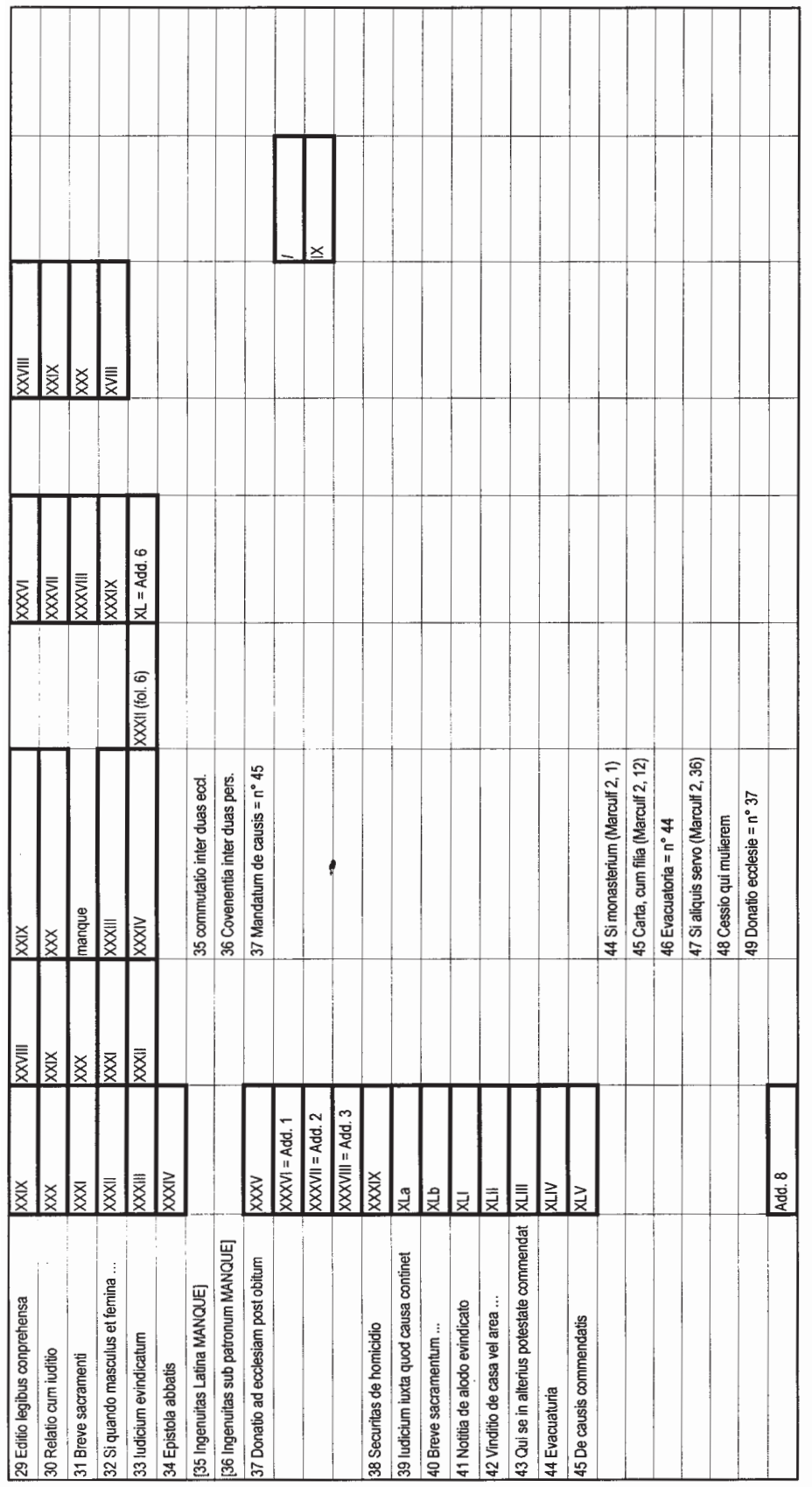


pelons que les formules "d'Angers ", " d'Auvergne " ou " de Sens " ne sont chacune connues que par un seul manuscrit et que les "Formules de Bourges " le sont par trois. Avec leurs cinq manuscrits presque complets (dont quatre ne témoignent pas d'un bouleversement majeur du recueil) et les quatre manuscrits comprenant seulement certains modèles d'actes, les "Formules de Tours " peuvent être comparées (sinon en notoriété, du moins en terme de nombre de copies) au Formulaire de Marculf, connu par sept manuscrits. Parmi l'ensemble des collections de formules, seul le recueil de Saint-Gall, réalisé par Notker le Bègue à la fin du IX siècle, a également connu un succès similaire : il figure dans six manuscrits.

On ne saurait trop plaider en faveur d'études prenant en compte les particularités de chaque manuscrit, considéré non plus comme le reflet d'une source originelle mais comme un document à part entière. De telles entreprises sont fondamentalement opposées à la recherche d'archétypes (selon la méthode de Karl Lachmann visant à l'établissement de l'Urtext) qui avait naguère guidé Karl Zeumer ${ }^{90}$, envers qui notre dette est au demeurant immense. Ce genre de travail fondé sur l'examen de la tradition manuscrite s'avère indispensable à une connaissance renouvelée de la diplomatique de l'acte privé et des conditions dans lesquelles les notaires pouvaient travailler. Soulignons par ailleurs que, eu égard au nombre assez conséquent de manuscrits contenant tout ou partie des " Formules de Tours ", il conviendrait de chercher de manière systématique les éventuelles influences que ces modèles ont pu exercer sur la rédaction des actes, notamment en Francie de l'Ouest. Il y aurait là matière à une enquête de grande envergure.

90. Cf. la citation supra, n. 30 . 


\section{RESUME}

Le formulaire élaboré à Saint-Martin de Tours au VIII ${ }^{\mathrm{e}}$ siècle comprend de nombreux modèles d'actes privés illustrant la diversité des occasions dans lesquelles on pouvait recourir aux scribes de ce grand monastère ligérien. Le nombre et la nature des manuscrits, dont certains s'avèrent de véritables recueils juridiques, nous permettent de penser que la diffusion (peut-être délibérément encouragée par les abbés, comme l'utilisation de ce formulaire dans la collection de Flavigny permet de le supposer) fut relativement large. On y reconnaît un indice supplémentaire de l'activité du scriptorium de SaintMartin.

\section{ABSTRACT}

The formulary made in Saint-Martin in the $8^{\text {th }}$ Century includes many models of private charters which show that the scribes of this great abbey of the Loire valley were requested to write documents in quite diverse occasions. The number and the contents of the manuscripts, some of them being real law-books, allow us to think that their diffusion (maybe deliberately promoted by the abbots, as suggested by the use of this formulary for making the Flavigny-collection) was relatively broad. One can see in this phenomenon an additional indication about the activity of the scriptorium of Saint-Martin. 
